\title{
An Efficient Simulated Annealing Algorithm for Network Reconfiguration in Large-Scale Distribution Systems
}

\author{
Young-Jae Jeon, Jae-Chul Kim, Member, IEEE, Jin-O. Kim, Member, IEEE, Joong-Rin Shin, Member, IEEE, and \\ Kwang Y. Lee, Fellow, IEEE
}

\begin{abstract}
This paper presents an efficient algorithm for loss minimization by using an automatic switching operation in largescale distribution systems. Simulated annealing is particularly well suited for a large combinatorial optimization problem since it can avoid local minima by accepting improvements in cost. However, it often requires a meaningful cooling schedule and a special strategy, which makes use of the property of distribution systems in finding the optimal solution. In this paper, we augment the cost function with the operation condition of distribution systems, improve the perturbation mechanism with system topology, and use the polynomial-time cooling schedule, which is based on the statistical calculation during the search. The validity and effectiveness of the proposed methodology is demonstrated in the Korea Electric Power Corporation's distribution system.
\end{abstract}

Index Terms-Combinatorial optimization problem, cooling schedule, distribution system, loss minimization, network reconfiguration, perturbation mechanism, power flow, simulated annealing.

\section{INTRODUCTION}

$\mathbf{E}$ FFICIENT operation of a distribution system can be achieved by reconfiguring the system to minimize system loss as the operating condition changes. The network reconfiguration problem essentially belongs to a combinatorial optimization problem since the problem is to determine open/closed status of all switches by considering all possible operational constraints in a large-scale distribution system. It is, therefore, difficult to obtain a true optimal solution fast in a real system.

Merlin and Back [1] first proposed a branch-and-bound method for distribution systems, which later was modified by Shirmohammadi and Hong [2]. Aoki et al. [3] used a

Manuscript received October 20, 2000; revised January 22, 2002. This work was supported in part by the Electrical Engineering and Science Research Institute under Grant 97-M-05 and by the National Science Foundation under Grant ECS-9705105.

Y.-J. Jeon and J.-C. Kim are with the Department of Electrical Engineering, Soongsil University, Seoul 156-743, South Korea (e-mail: anneal@dreamwiz.com; jckim@ee.ssu.ac.kr).

J.-O. Kim is with the Department of Electrical Engineering, Hanyang University, Seoul 133-791, South Korea (e-mail: jokim@hanyang.ac.kr).

J.-R. Shin is with Department of Electrical Engineering, Kon-Kuk University, Seoul 143-701, South Korea (e-mail: jrshin@konkuk.ac.kr).

K. Y. Lee is with Department of Electrical Engineering, Pennsylvania State

University, University Park, PA 16802 USA (e-mail: kwanglee@ psu.edu).

Digital Object Identifier 10.1109/TPWRD.2002.803823 quasiquadratic nonlinear programming technique to minimize the distribution system loss. Civanlar et al. [4] and Baran and Wu [5] proposed approximate power-flow methods for loss minimization, resulting from a switch operation in distribution systems. Liu et al. [6] used an expert system technique to solve the reconfiguration problem for distribution systems. Chiang and Jean-Jumeau [7], [8] proposed a solution procedure for the reconfiguration problem using simulated annealing, which later was extended by Chang and Kuo [9], Jiang and Baldick [10], and Su and Lee [11]. Nara et al. [12] implemented the genetic algorithm to find the minimum loss configuration.

Although the branch-and-bound, branch-exchange, and expert system techniques can solve the problem with rather less computational burden, the calculated results are only approximates and local optima. Moreover, although other heuristic methods work well in a small system, it is difficult to find global optimum in a real system that would have a large number of switches. Recently, a genetic algorithm and tabu search were used in combinatorial optimization problems [12]-[15]. A genetic algorithm generates new solution candidates through crossover and mutation of strings, but many infeasible solutions that violate the radial configuration are generated. Tabu search generally finds a good solution, but it does not have a good convergence property. Because of its flexible nature, tabu search would be better in hybrid with another algorithm rather than as an independent application.

Although the methods mentioned before do not have a good convergence property in comparison with simulated annealing, they are used due to less computation time. Distribution systems are growing continually and becoming more complex. However, computer technology has advanced remarkably. Therefore, simulated annealing is particularly well suited for reconfiguration problems in large-scale distribution systems. However, simulated annealing requires an elaborate cooling schedule and a special strategy to find the optimal solution in large-scale distribution systems. In this paper, we introduce the polynomial-time cooling schedule, which is based on the statistical calculation during the search. A novel strategy is also used to make the cost function "landscape" smooth. Moreover, a new perturbation mechanism is developed to generate a network configuration by considering the system topology. The proposed procedure avoids local minima better in the complex solution surface.

The rest of the paper is organized as follows: In Section II, the problem formulation and distribution system power flow are presented. In Section III, the essence of the simulated annealing 


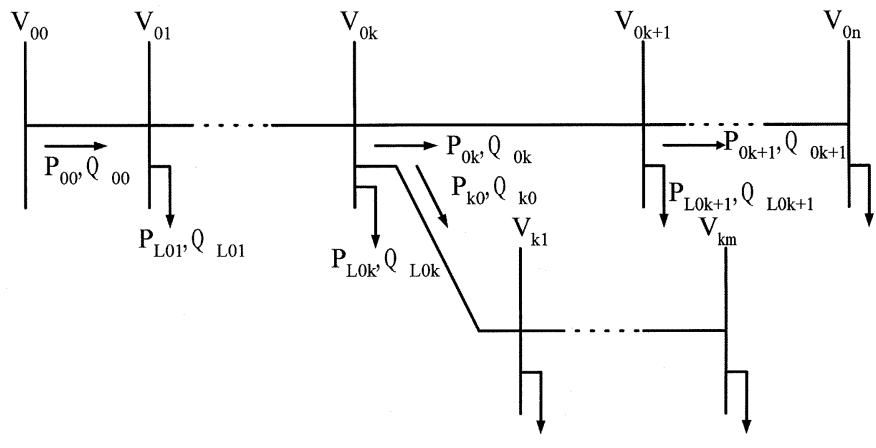

Fig. 1. One-line diagram of a main feeder with laterals.

is presented, and a detailed solution methodology through simulated annealing is explained in Section IV. In Section V, numerical examples are shown to demonstrate the validity and effectiveness of the proposed methodology, and conclusions are drawn in Section VI.

\section{PROBLEM Formulation}

The network reconfiguration problem in a distribution system is to find a configuration with the minimum power loss while all system constraints are satisfied. In this paper, we use the power-flow method of Baran and $\mathrm{Wu}$ [5] to determine the power flow approximately in a radial distribution system as shown in Fig. 1.

Due to the complexity of a large-scale distribution network, the network reconfiguration problem normally assumes a symmetrical systems and constant loads. Therefore, the distribution lines are modeled as series impedances $Z_{l}=r_{l}+j x_{l}$. Load demand at bus $k$ is modeled as a constant and balanced power sink $S_{L k}=P_{L k}+j Q_{L k}$. The real and reactive power flow at the receiving end of branch $k+1, P_{k+1}$, and $Q_{k+1}$, and the voltage magnitude at the receiving end, $\left|V_{k+1}\right|$, can be expressed by the following set of equations:

$$
\begin{aligned}
P_{k+1} & =P_{k}-P_{L k+1} \\
Q_{k+1} & =Q_{k}-Q_{L k+1} \\
V_{k+1}^{2} & =V_{k}^{2}-2\left(r_{k+1} P_{k}+x_{k+1} Q_{k}\right) .
\end{aligned}
$$

Equations (1)-(3) are known as the Simplified DistFlow equations. They are not accurate in comparison with the DistFlow equations [5], but the calculation error is acceptable in view of the negligible error in the section load estimation.

In the distribution system power-flow equations, several boundary conditions must be satisfied:

1) at the substation, the voltage magnitude $\left|V_{0}\right|$ is given;

2) at the end of the main feeder: $P_{0 n}=0$ and $Q_{0 n}=0$;

3) at the end of lateral $k: P_{k m}=0$ and $Q_{k m}=0$

where $n$ and $m$ are the node numbers of the main feeder and branch $k$, respectively.

The power loss in the distribution system can be calculated as the sum of the $i^{2} r$ loss in each branch. The total power loss can be calculated by

$$
P_{\text {loss }}^{\text {total }}=\sum_{i=1}^{l} r_{i}\left(P_{i}^{2}+Q_{i}^{2}\right)
$$

where $l$ is the total number of branches.

\section{Simulated ANNEALING}

In statistical mechanics, a physical process known as annealing is often performed in order to relax the system to a state with minimum free energy. In the annealing process, a solid in a heat bath is heated up by increasing the temperature of the bath until the solid melts into liquid, then the temperature is lowered slowly. In the liquid phase, all particles of the solid arrange themselves randomly. In the ground state, the particles are arranged in a highly structured lattice and the energy of the system is minimal. The ground state of the solid is obtained only if the maximum temperature is sufficiently high and the cooling is performed sufficiently slow. Otherwise, the solid will be frozen into a metastable state rather than into the ground state. If a state is defined by the set of particle positions, then, at thermal equilibrium, the probability of the system being in state $\mathrm{i}$ is represented by the Boltzman distribution [16], [17],

$$
\pi_{i}=\operatorname{Pr}\{s=i\}=\frac{\exp \left(-E(i) / k_{b} T\right)}{Z}
$$

where $Z=\Sigma_{i \in S} \exp \left(-E(i) / k_{b} T\right)$ is known as the partition function, $k_{b}$ is the Boltzman constant, $T$ is the temperature, $E(i)$ is the energy of the state $i$, and $S$ is the state space [16], [17]. At a very high temperature, it can be seen that

$$
\lim _{T \rightarrow \infty} \pi_{i}=\lim _{T \rightarrow \infty} \frac{\exp \left(-E(i) / k_{b} T\right)}{\sum_{j \in S} \exp \left(-E(j) / k_{b} T\right)}=\frac{1}{|S|}
$$

where $|S|$ denotes the total number of states in $S$. This implies that all of the states are equally probable at a very high temperature. In this case, the energy of the state does not affect the probability of the state. On the other hand, at a lower temperature, the value of exponential function is strongly affected by the energy of the state. Therefore, we have

$$
\begin{aligned}
\lim _{T \rightarrow 0} \pi_{i} & =\lim _{T \rightarrow 0} \frac{\exp \left(-\left(E(i)-E_{\min }\right) / k_{b} T\right)}{\sum_{j \in S} \exp \left(-\left(E(j)-E_{\min }\right) / k_{b} T\right)} \\
& = \begin{cases}\frac{1}{\left|S_{\min }\right|}, & \text { if } i \in S_{\min } \\
0, & \text { otherwise }\end{cases}
\end{aligned}
$$

where $S_{\min }=\left\{i: E(i)=E_{\min }\right\}$ and $E_{\min }=\min _{j \in S} E(j)$. From this equation, we observe that as the temperature approaches zero, the system will converge to the state with the minimum energy, $E_{\min }$, since the probability of the state only with the minimum energy is nonzero, while the probability of others is 0 .

To illustrate this concept, consider the generalized Boltzman distribution of a simple system with energy function $f(x)=$ $x^{2}$, shown in Fig. 2. This system has nine states between -2.0 and 2.0 with intervals of 0.5 , and its Boltzman distribution is illustrated for $T=99.0,1.0$, and 0.1 .

In Fig. 2, it can be seen that all of the states are equally probable at $T=99.0$, while the probability of the state with minimum energy is very high at $T=0.1$. Therefore, if the system follows the Boltzman distribution, the state with minimum energy can be obtained by decreasing the temperature.

Based on the annealing process in the statistical mechanics, the simulated annealing was independently introduced for 


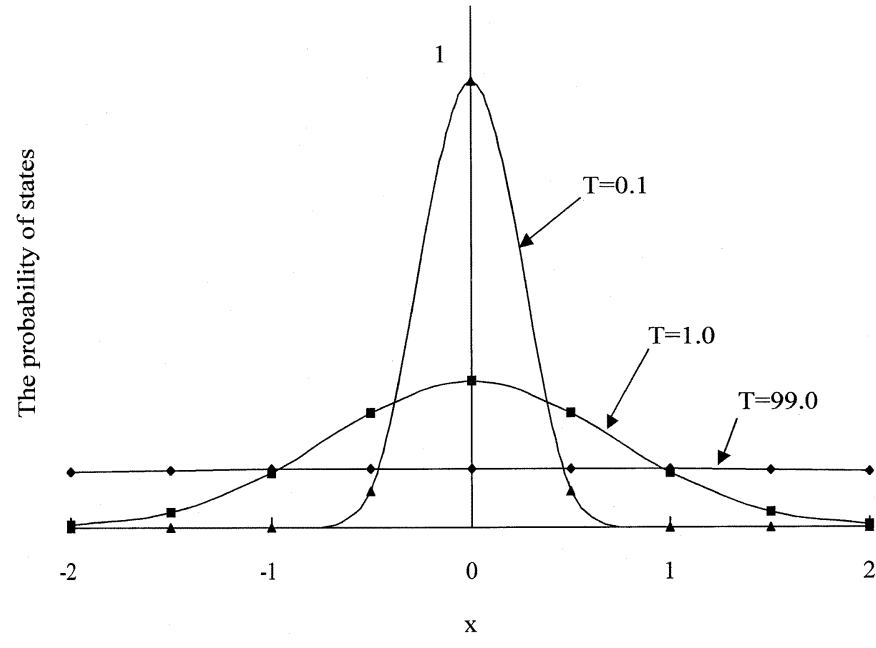

Fig. 2. Boltzman distribution with different temperatures.

TABLE I

Analogy Between Physical System and Simulated AnNealing

\begin{tabular}{c|c}
\hline Physical System & Optimization Problem \\
\hline State & Solution (Configuration) \\
\hline Energy & Cost \\
\hline Ground State & Optimal. Solution \\
\hline Rapid Quenching & Local Search \\
\hline Careful Annealing & Simulated Annealing \\
\hline
\end{tabular}

solving complicated combinatorial optimization problems by Kirkpatrick et al. in 1983 and Cerny in 1985 [18], [19]. The name "simulated annealing" originates from the analogy with the physical process of solids. The analogy between physical system and simulated annealing is tabulated in Table I.

As shown in Table I, the cost function and the solution (configuration) in the optimization process correspond to the energy function and the state of statistical physics, respectively.

Suppose that a cost function $f: S \rightarrow R^{+}, s \in S$, to be minimized is dined on some finite set $S$. In simulated annealing, given the current state $s(k)$, a neighboring state $s^{\prime}(k)$ is randomly selected from a neighboring set $N(s)$, where $k$ is the $k$ th trial. The transition probability from state $s(k)$ to $s^{\prime}(k)$ is given by the Metropolis criterion [16], [17]

$$
P\left[s(k), s^{\prime}(k)\right]=\exp \left[\frac{-\left[f\left(s^{\prime}(k)\right)-f(s(k))\right]}{T}\right] .
$$

From (8), it can be seen that the Metropolis criterion, while performing the local search for the minimum cost at a fixed temperature $T$, enables occasional transition from a lower-cost configuration to a higher-cost configuration with certain probability, thus preventing the system from getting stuck in a local minimum. The random process can be characterized by a discrete-time Markov chain [16], [17]. Under the feature of Markov chain, the stationary equilibrium distribution $\pi_{i}$ for configuration $i$ exists after an infinite number of transitions.

$$
\pi_{i}(T)=\frac{\exp (-f(i) / T)}{\sum_{j \in S} \exp (-f(j) / T)} .
$$

From (7), we know that

$$
\pi_{i}^{*}=\lim _{T \rightarrow 0} \pi_{i}(T)= \begin{cases}\frac{1}{\left|S_{\min }\right|}, & \text { if } i \in S_{\min } \\ 0, & \text { otherwise. }\end{cases}
$$

Therefore,

$$
\begin{aligned}
\lim _{T \rightarrow 0}\left[\lim _{k \rightarrow \infty} P\left(s(k) \in S_{\min }\right)\right] & =\lim _{T \rightarrow 0} \sum_{i \in S_{\min }} \pi_{i}(T) \\
& =\sum_{i \in S_{\min }} \pi_{i}^{*}=1 .
\end{aligned}
$$

Equation (11) states that the simulated annealing asymptotically converges to configurations with the minimum cost (i.e., if the temperature is slowly lowered and at each temperature the system performs a sufficient number of transitions, the configurations with the global minimum cost can be found with probability one [16], [17], [20].

\section{SOLUTION ALGORITHM}

In a large combinatorial optimization problem, an appropriate perturbation mechanism, cost function, solution space, and cooling schedule are required to find an optimal solution with simulated annealing. In this section, these elements for the network reconfiguration problem in distribution systems are revisited and their improvements are proposed.

\section{A. Topology-Based Perturbation Mechanism}

The open/closed status of sectionalizing switches and tie switches determines the network configuration of the system. Hence, a new system configuration can be generated from current system configuration with a perturbation mechanism that changes the status of sectionalizing and tie switches. To achieve a new system configuration, the add/subtract perturbation mechanism proposed by Chiang and Jean-Jumeau [7], [8] is described as follows.

1) Randomly choose a switch $\boldsymbol{t}$ from a tie switch set $\Omega_{t i e}$, and then close it. The switch $t$ is removed from $\Omega_{t i e}$ and placed in a sectionalizing switch set $\Omega_{\mathrm{sec}}$. This creates a loop in the system, and sectionalizing switches in the loop are included in a loop set $\Omega_{\text {loop }}$

2) Randomly choose a switch from $\Omega_{\text {loop }}$ and then open it. This will restore the system back to a radial structure. The switch s is removed from the set $\Omega_{\mathrm{sec}}$ and added to the set $\Omega_{\text {tie. }}$.

This perturbation mechanism is illustrated using the simple radial distribution system as shown in Fig. 3 [5]. Tie switches and sectionalizing switches represent the switch on the dotted lines and solid lines, respectively. When closing the tie switch 34 , a loop 2 is created and sectionalizing switches $9-14$ are included in $\Omega_{\text {loop }}$. If any sectionalizing switch in $\Omega_{\text {loop }}$ is opened, the system configuration is restored to a radial structure. As a result of this switching, a new system configuration can be generated, which satisfies the radial configuration and power-supply constraints.

This perturbation mechanism is suitable for the network reconfiguration problem, but its drawback is that it is not taking advantage of knowing the system topology. Tie and 


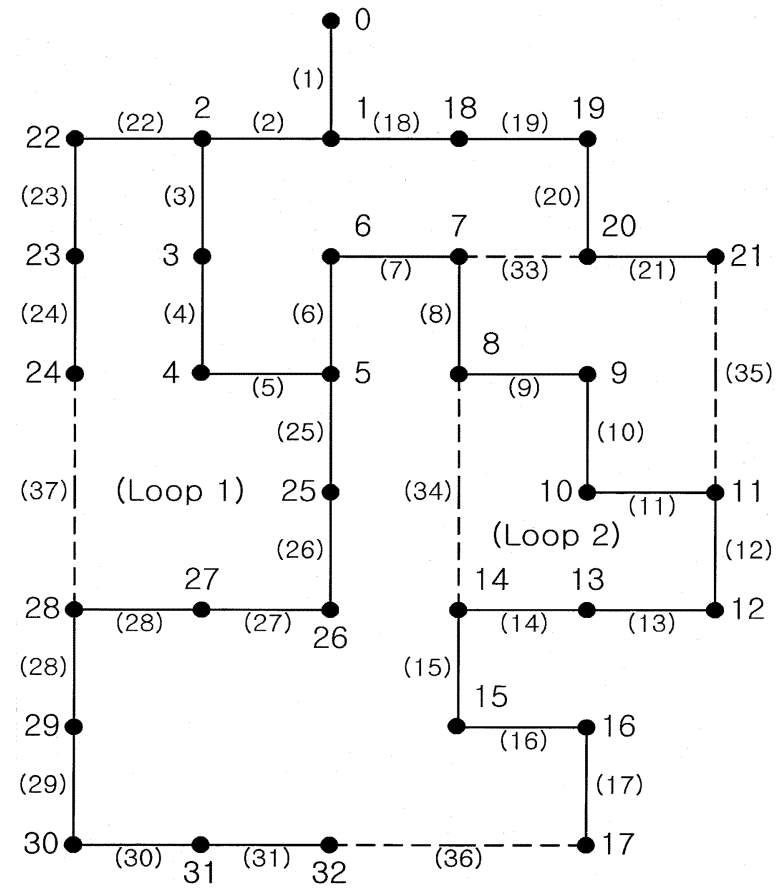

Fig. 3. Simple radial distribution system.

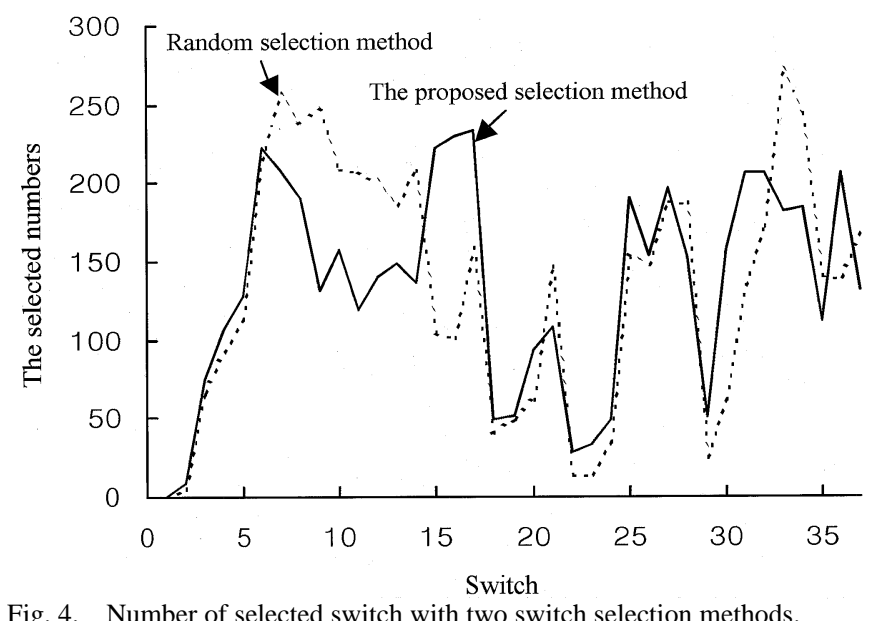

Fig. 4. Number of selected switch with two switch selection methods.

sectionalizing switches that are randomly selected implies that system topology and temperature are not affecting the search process and, therefore, the search property of simulated annealing cannot be influenced.

As shown in Fig. 3, the local solution space of loop 1 is larger than that of loop 2 due to the different size of the loops. If all of the tie switches are selected with equal probability, in loop 1 the search may not be performed sufficiently. On the other hand, in loop 2, the search often returns to a configuration just visited because the search is performed more than what is necessary. Also, it is necessary that tie switches are selected in relation to the size of a loop because the size of each loop is significantly different in large-scale distribution systems. For example, when the length of Markov chain is 100 and temperature is decreased 50 times by decrement function (i.e., for which the perturbation mechanism generates 5000 solutions), the selected switches are shown in Fig. 4.
In Fig. 4, the switches in loop 2, which are 9-14, are selected less often by the proposed selection method than by the random selection method, while switches in other larger loops are selected more often. The proposed selection method induces diversity in the search.

In Fig. 3, if the tie switch 37 is closed, any sectionalizing switch in loop 1 must be opened in order to remain as a radial structure. If the sectionalizing switch 3 is opened, the system may be largely perturbed, which can be accepted by the Metropolis criterion at high temperature, although the cost may be increased. However, most of these largely perturbed transitions must be rejected at a lower temperature. Therefore, it is necessary that a perturbation mechanism generates an acceptable configuration at a low temperature. From these observations, the following topology-based perturbation mechanism (TPM) is proposed to select tie and sectionalizing switches.

1) Given an initial radial configuration and a set of tie switches, for each tie switch there exists a loop formed by the closing of the switch. Then, the size of the loop (i.e., the number of sectionalizing switches in the loop) can be determined. Determine the size of the loops corresponding to all tie switches. Normalize each loop size with the sum of all loop sizes. The result is the relative loop size.

2) Perform a size-proportionate selection of a tie switch through the simulated spin of a weighted roulette wheel. The roulette wheel is biased with the relative loop sizes that correspond to each tie switch. Close the selected tie switch, creating a loop in the system. Form the loop set $\Omega_{\text {loop with }}$ all of the sectionalizing switches in the loop. This forces tie switches selected in relation to the size of the loop.

3) For all sectionalizing switches in $\Omega_{\text {loop }}$, calculate the "switch level," (i.e., the distance between the tie switch $\mathrm{t}$ and each sectionalizing switch). Choose and open a sectionalizing switch with the switch-level-dependent selection probability in $\Omega_{\text {loop }}$. This will restore the system back to a radial structure.

The selection probability of sectionalizing switches is determined by the uniform distribution biased with the inverse of the switch level

$$
S W_{i}=\frac{1}{n} \gamma_{k}+\left(1-\gamma_{k}\right) \frac{1 / S L_{i}}{\sum_{j=1}^{n} 1 / S L_{j}}
$$

where $S W_{i}$ and $S L_{i}$ are the respective selection probability and switch level of the sectionalizing switch $i, n$ is the number of sectionalizing switches in $\Omega_{\text {loop }}$, and $\gamma_{k}$ is a positive number in the range $[0,1]$, which is calculated by the following equation:

$$
\gamma_{k}=\lambda+\lambda e^{\left(T_{k}-T_{0}\right)}
$$

where $\lambda$ is a positive number in the range [0, 0.5], and $T_{0}$ and $T_{k}$ are the initial and current temperatures, respectively.

At $T_{0}$ and with $\lambda=0.5$, all sectionalizing switches are randomly selected (i.e., $\gamma_{k}$ is 1 ), and each sectionalizing switch may be selected by the same probability. By decreasing the temperature $T_{k}, \gamma_{k}$ is decreased, which increases the selection probability of the switch with the small switch level. This implies that 


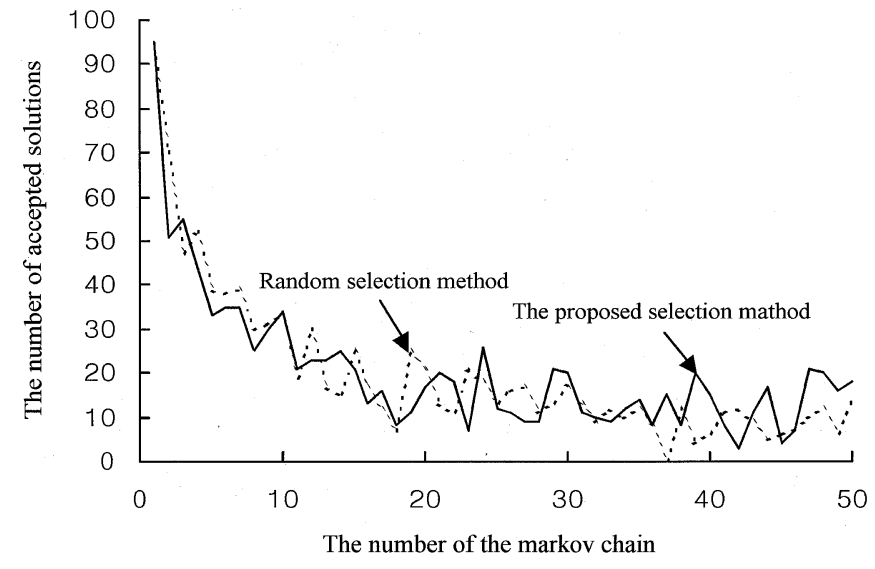

Fig. 5. Number of accepted solution with two perturbation mechanisms.

a slightly perturbed configuration from the proposed topologybased perturbation mechanism is quite probable, which can be easily accepted as a new configuration at low temperature.

As an example for the selection of sectionalizing switches, when the length of Markov chain is 100 and temperature is decreased 50 times, the numbers of accepted solutions by the Metropolis criterion are shown in Fig. 5.

As shown in Fig. 5, the proposed topology-based perturbation mechanism generates more new solutions than the random perturbation mechanism. The proposed and random perturbation mechanism generates 1015 and 989 solutions for 5000 trials, respectively, and 317 and 252 solutions from the 26th to 50th Markov chain, respectively. This implies that a slightly perturbed configuration from the proposed topology-based mechanism is quite probable, which can be easily accepted as a new configuration at low temperature.

The proposed topology-based perturbation mechanism changes the status of tie and sectionalizing switches with the system topology and temperature, which is diversified at a high temperature and intensified at attraction regions at lower temperature.

\section{B. Cost Function and Solution Space}

In the network reconfiguration problem, the solution space is the set of all possible open/closed status of the switches. The new system configuration is generated by using the proposed topology-based perturbation mechanism, but it is difficult to satisfy all of the network reconfiguration constraints. In this paper, the set of network reconfiguration constraints is divided into two subsets to generate efficiently a network configuration satisfying all network reconfiguration constraints. Any violation of power-supply and radial configuration constraints can be avoided by using the perturbation mechanism before the power-flow calculation, while any violation of the line-capacity and voltage-drop constraints is penalized through penalty factors after the power-flow calculation. In this paper, we will call the former and latter as "before" and "after" constraints, respectively. The set of network reconfiguration constraints are as follows:

"Before" constraints: $C_{0}=\{$ Power Supply, Radial Configuration\}

"After" constraints: $C_{1}=\{$ Line Capacity, Voltage Drop $\}$
Thus, the set of solutions deemed feasible by the simulated annealing is defined as

$$
X=\left\{x \mid x \text { satisfies all constraints in } C_{0}\right\} .
$$

The network reconfiguration problem is to minimize the total system loss (4) while satisfying the "after" constraints. That is,

Minimize $f(x)=\left(P_{\text {loss }}^{\text {total }}+\alpha A(x)+\beta B(x)\right), \quad x \in X$

where $\alpha$ and $\beta$ are penalty factors and $A(x)$ and $B(x)$ correspond to the set of violations to line capacity and voltage drop constraints, respectively.

In the cost function, line capacity and voltage drop constraints are included by using the penalty factors because these constraints can be checked for violation after the power-flow calculation. When any solution violates constraints, this infeasible solution is generally discarded or its selection probability is decreased by using penalty factors. Solving by using this method is always feasible and the size of the solution space is decreased. However, in a large combinatorial optimization problem, it is difficult to find an optimal solution because the solution surface is very complex and multimodal. Also, if an optimal solution must pass through an infeasible solution space, it may not find the optimal solution.

If a slightly infeasible solution is included in the solution space by using small penalty factors, the solution is easily generated and the solution surface is smoothed. However, the size of the solution space is generally increased and it is necessary that any fine-tuning algorithm should turn from an infeasible solution to a feasible solution.

In this paper, we incorporate these features. The line-capacity constraint is a sensitive issue in the network reconfiguration of a real distribution system because line-capacity constraints are composed of many varieties of lines. Although a planner designs a distribution system by considering this issue, the violation of a line-capacity constraint in large-scale distribution systems is rather common and more than in small distribution systems during the optimization process. Therefore, in this paper, penalty factors are adjusted by multiplying $1 / T_{k}$, where $T_{k}$ is the temperature. In the beginning of the search, small penalty factors lead to contain a slightly infeasible solution. As the temperature decreases, penalty factors are increased and only feasible solutions are generated at the latter part of the search.

As shown in Fig. 6, the presence of slightly infeasible solutions leads to a smoothing of the cost function "landscape," which enables the simulated annealing to escape more easily from local minima and to reach rapidly in the vicinity of an optimal solution. Penalty factors are increased with decreasing temperature, and the infeasible solution will be excluded at the end. Therefore, with the new definition of cost (15) and the smoothing strategy, all trial solutions are made feasible and the search is efficiently performed.

\section{Polinomial-Time Cooling Schedule}

If the temperature is slowly lowered and at each temperature the system performs the infinite number of transitions, the configuration with the global minimum cost can be found. But 


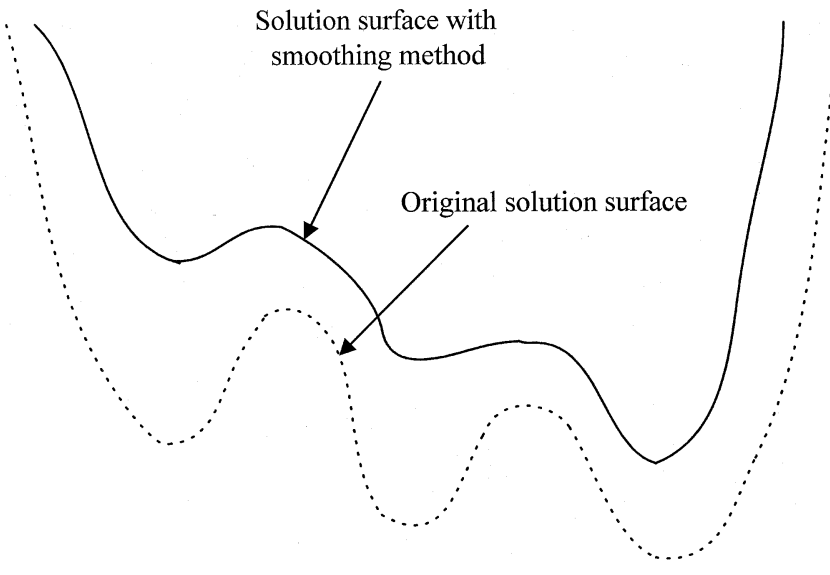

Fig. 6. Solution surface with the smoothing strategy.

the infinite number of transitions is impossible in the real simulation. Therefore, a finite-time implementation of the simulated annealing is realized by generating Markov chains of finite length for a finite sequence of descending temperatures. To do this, a set of parameters that govern the convergence of the algorithm is specified. These parameters form a cooling schedule, which is defined as following:

- a finite sequence of the temperature, (i.e.,

- an initial temperature $T_{0}$;

- a decrement function for decreasing temperature;

- a final temperature specified by a stop criterion);

- a finite number of transitions for each temperature (i.e.,

- a finite length for each Markov chain).

In this paper, we use the polynomial-time cooling schedule proposed by Aarts and Laarhoven in [17]. This cooling schedule leads to a polynomial-time execution of the simulated annealing, but it cannot guarantee the optimal solution [16], [17], [21]. Different parameters of the cooling schedule are determined based on the statistics calculated during the search. In the following, we describe these parameters.

1) Initial Temperature $T_{0}$ : Initial temperature $T_{0}$ should be large enough to support virtually all transitions to be accepted. This is achieved by requiring that the acceptance ratio $(A R)$, the ratio accepting transitions in $m$ trials, is close to one. Assume that a sequence of $m$ trials is generated at a certain temperature $T$. Let $m_{1}$ denote the number of transitions from $i$ to $j$ for which $f(i) \geq f(j)$, and $m_{2}$ is the number of transitions for which $f(i)<f(j)$. Furthermore, let $\overline{\Delta f}^{(+)}$be the average difference in cost over the $m_{2}$ cost-increasing trials. Then $A R$ can be approximated by the following equation [17]:

$$
A R \approx \frac{\left(m_{1}+m_{2} \cdot \exp \left(-\overline{\Delta f}^{(+)} / T\right)\right)}{\left(m_{1}+m_{2}\right)}
$$

from which we obtain

$$
T=\frac{\overline{\Delta f}^{(+)}}{\ln \left(m_{2} A R-m_{1}(1-A R)\right)} .
$$

The temperature $T_{0}$ can be calculated the following way: Initially, $T$ in (16) is set to zero. Next, a sequence of $m$ trial is generated, and $m_{1}, m_{2}, A R$, and $\overline{\Delta f}^{(+)}$are obtained, where $m=m_{1}+m_{2}$. A new temperature is determined using (17). Equations (16) and (17) are recursively calculated until $A R$ reaches a previously specified value. A final temperature $T$ obtained in this way is then taken as the initial temperature $T_{0}$.

2) Decrement Function of the Temperature $T_{k+1}$ : The length of Markov chain and decrement function for changing $T_{k}$ into $T_{k+1}$ are strongly related through the concept of quasiequilibrium. If decrement in $T_{k}$ is large, it takes longer to establish quasiequilibrium at $T_{k+1}$. Thus, there is a tradeoff between fast decrement of $T_{k}$ and the length of Markov chain. The value $T_{k+1}$ is related to the current value, $T_{k}$, by the following function [17]:

$$
T_{k+1}=\frac{T_{k}}{1+\frac{T_{k} \ln (1+\delta)}{3 \sigma_{T_{k}}}}
$$

where $\sigma$ is the standard deviation of the cost values generated in $T_{k}$, and $\delta$ is a constant called the distance parameter. Small $\delta$ values lead to small decrements in $T_{k}$.

3) Final Temperature: Termination in this schedule is based on an extrapolation of the expected average cost at the final temperature. Hence, the algorithm is terminated if for some value of $k$ we have

$$
\left.\frac{T_{k}}{\langle f\rangle_{\infty}} \cdot \frac{\partial\langle f\rangle_{T}}{\partial T}\right|_{T=T_{k}}<\varepsilon
$$

where $\langle f\rangle_{\infty} \approx\langle f\rangle_{T_{0}}$ is the average cost at initial temperature $T_{0},\langle f\rangle_{T_{k}}$ is the average cost at the $k$ th Markov chain, $\partial\langle f\rangle_{T} /\left.\partial T\right|_{T=T_{k}}$ is the rate of change in the average cost at $T_{k}$, and $\varepsilon$ is a positive number. We refer to $\varepsilon$ as the stopping parameter and (19) as the stopping criterion.

4) Length of Markov Chains: In [16], [17], it is concluded that the decrement function of the temperature, (18), requires only a "small" number of trial solutions to rapidly approach the stationary distribution for a given temperature. In general, a chain length of more than 100 transitions is reasonable.

\section{Solution Algorithm for Network Reconfiguration}

In conclusion, the network reconfiguration methodology by using simulated annealing is summarized in detail as follows.

Step 1): Input system data and initialize parameters,

input the system and network data, initialize the current solution $x_{0}$, the optimal solution $x_{o p t}=x_{0}$, acceptance ratio, and the length of Markov chain, and determine initial temperature $T_{0}$ using (16) and (17).

Step 2): Generate the updating term of solution $x$.

A new configuration is generated by the proposed topologybased perturbation mechanism.

Step 3): Calculate the cost difference $\Delta f=f(x+\Delta x)-$ $f(x)$.

Calculate the cost difference by (15); line capacity and voltage drop constraints are penalized by penalty factors.

Step 4): Check for $\Delta f<0$.

If $\Delta f<0$, go to Step 6). Otherwise, go to Step 5).

Step 5): Check the Metropolis criterion.

If $\exp \left(-\Delta f / T_{k}\right)>R$, where $R$ is a random number in the range $[0,1]$, then go to Step 6$)$. Otherwise, restore to the previous configuration and go to Step 2). 
Step 6): Update the system configuration.

Retain the new configuration. If the number of perturbations is not less than the length of Markov chain, go to Step 7). Otherwise, go to Step 2).

Step 7): Reduce the temperature.

Calculate the standard deviation of the cost at the $k$ th Markov chain, and then calculate the new temperature $T_{k+1}$ with (18).

Step 8): Check the stopping criterion.

If (19) is satisfied, stop. Otherwise, continue the process by returning to Step 2).

\section{NUMERICAL RESULT}

Application of the simulated annealing generally requires specification of three items: i) a concise problem representation, ii) a perturbation mechanism, and iii) a cooling schedule. In addition, two additional items are required according to our experience. One is to make the cost function small. If the cost is small, the exponential function is sensitive for the small changes in the cost, which results in good moves in the search process. If the cost is large, a randomly performed search takes longer. Another is to select a good random number generator. Theory of simulated annealing assumes a true random number generator, but the rand() function in a compiler generally has a short-cycle period. Use of the random number generator with a long cycle period is desirable for a large-scale optimization problem. In this paper, the random number generator proposed by Kirkpatrick and Stoll is used [22]. These items are often neglected when applying the simulated annealing. However, they are important to produce the best results. Considering the items just mentioned, the proposed methodology was demonstrated in various distribution systems.

The proposed methodology was implemented in $C$ language on an Intel Pentium II $350-\mathrm{MHz}$ processor with a double linked-list data structure to represent the data base of the distribution system. The proposed methodology is tested in several systems, including a 148-bus system and a real distribution system of the Korea Electric Power Corporation (KEPCO).

\section{A. The 148-Bus System}

The 148-bus system is part of a real distribution system in Joongdong City, a satellite city of Seoul, Korea. The rated voltage is $22.9 \mathrm{kV}$ and total load is $44.43 \mathrm{MW}$ and 21.51 MVAR. The system consists of 148 buses, 148 sectionalizing switches, and 19 tie switches as shown in Fig. 7. The dot "(•)" in Fig. 7 denotes a bus and the switches are connected between the buses. The switches between the following buses are opened in the initial configuration: (41-42), (24-56), (38-59), (9-60), (47-66), (54-75), (61-78), (74-95), (68-98), (102-103), (105-113), (106-120), (119-127), (101-136), (28-142), (96-143), (72-144), (49-147), and (145-148).

In the initial configuration, the total power loss and the minimum bus voltage of the system is $1068 \mathrm{~kW}$ and 0.935 p.u., respectively.

Fig. 8 is the final result of the simulation with the proposed methodology. Optimal configuration has a total power loss of $860 \mathrm{~kW}$ and minimum bus voltage of 0.953 p.u. The switches connecting the following buses are opened for the

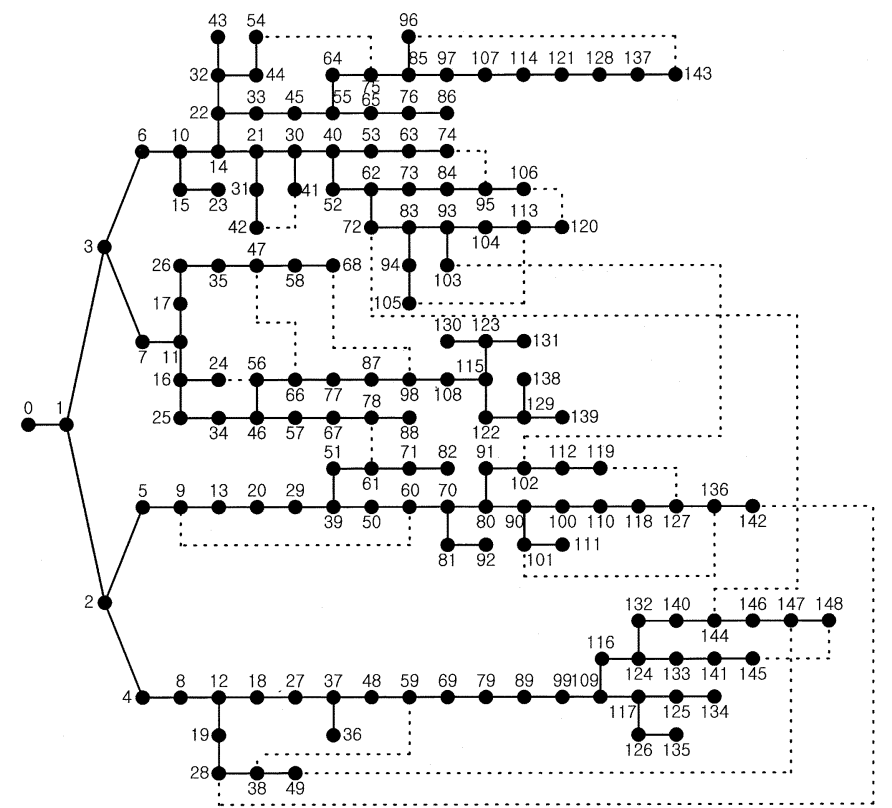

Fig. 7. Initial configuration of the KEPCO 148-bus system.

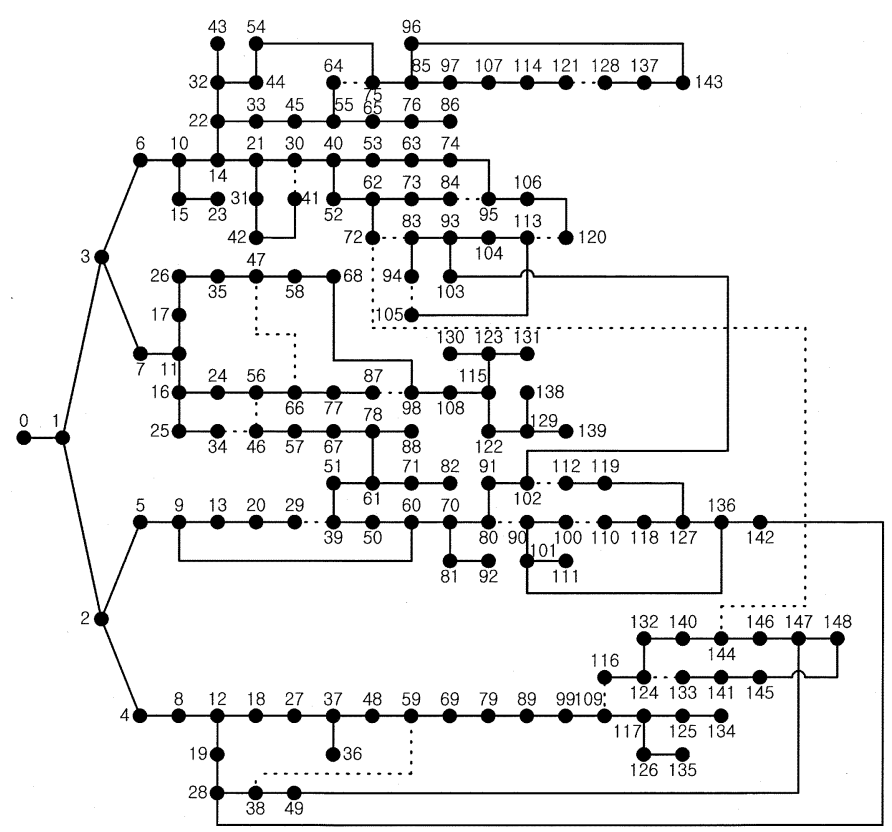

Fig. 8. Optimal configuration of the KEPCO 148-bus system.

optimal configuration: (29-39), (30-41), (34-46), (46-56), (38-59), (47-66), (64-75), (72-83), (80-90), (84-95), (87-98), (94-105), (100-110), (102-112), (109-116), (113-120), (121-128), (124-133), and (72-144). In the implementation, good results have been reached with the parameters $\alpha=1000.0, \beta=1000.0$, acceptance ratio $=0.6$, the length of Markov chain $=100, \lambda=0.5, \delta=2.0$, and $\varepsilon=0.5$.

The proposed methodology is tested on a 32-bus [5], 69-bus [8], and the 148-bus systems and compared with a conventional simulated annealing algorithm, which is performed with the cooling schedule by Kirkpatrick et al. [16], [18]. In the conventional simulated annealing, good results have been reached with the parameter $\alpha=1000.0, \beta=1000.0$, acceptance 


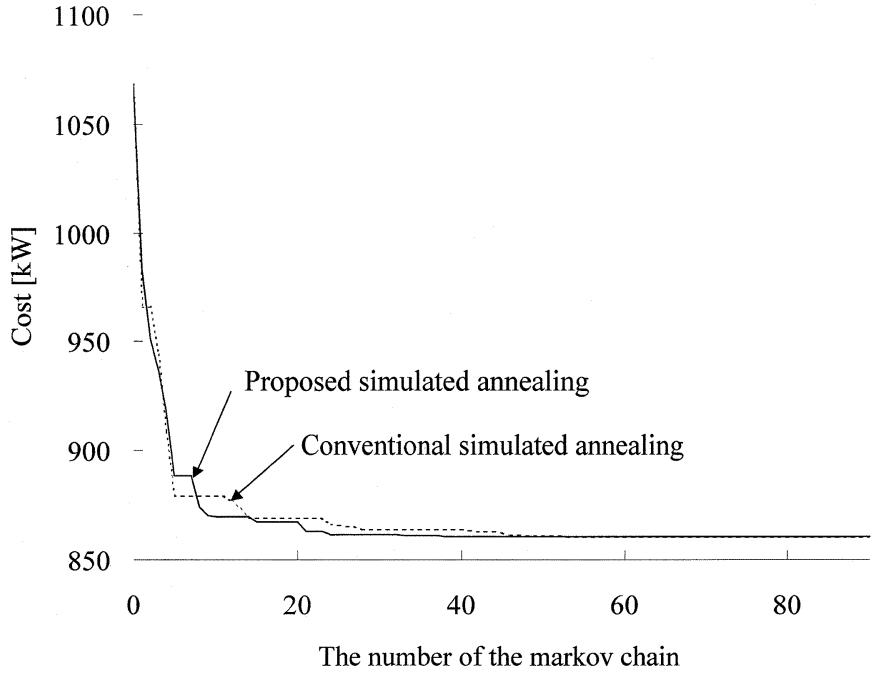

Fig. 9. Convergence properties of two simulated annealing algorithms for the 148-bus system.

TABLE II

COMPARISON OF CONVENTIONAL AND PROPOSED SAS

\begin{tabular}{c|c|c|c}
\hline System & 32 bus[5] & 69 bus[8] & 148 bus \\
\hline Conventional SA & $0.30[\mathrm{sec}]$ & $250[\mathrm{sec}]$ & $74.64[\mathrm{sec}]$ \\
\hline Proposed SA & $0.34[\mathrm{sec}]$ & $2.57[\mathrm{sec}]$ & $68.44[\mathrm{sec}]$ \\
\hline
\end{tabular}

ratio $=0.85$, decrement constant $=0.9$, the length of Markov chain $=100$, and final value in the stopping criterion $=30$. The convergence profile is shown in Fig. 9 and the results of the comparisons are tabulated in Table II for the 32-, 69-, and 148-bus systems, where the computation time of both methods is for the average of ten trials for a fair comparison.

In the 32- and 69-bus systems, an optimal solution can be easily obtained by small transitions, and both methods have comparable results in computation time. In the 148-bus system, the conventional and the proposed simulated annealing methods yielded the same system loss and the minimum voltage drop. However, the percentage reduction in the computation time is approximately $10.7 \%$ by the proposed simulated annealing method. The proposed methodology used meaningful cooling schedule and a perturbation mechanism by considering the system topology and temperature, and is shown to be efficient in middle or large distribution systems.

\section{B. KEPCO's Real Distribution System}

This system is the real distribution system in Joongdong City, a satellite city of Seoul, Korea. The rated voltage is $22.9 \mathrm{kV}$. There are one substation, four main transformers, 25 feeders, 362 buses, and 421 switches in the distribution system. This system is mostly composed of underground cable (CN-CV 325 $\mathrm{mm}^{2}$ ), and the total loads are $150 \mathrm{MW}$ and $75 \mathrm{MVAR}$.

The initial configuration is altered to change the status of many tie switches because the original configuration is considered to be near optimal. In the initial configuration, total power loss and the minimum bus voltage of the system is $3959 \mathrm{~kW}$ and 0.9 p.u., respectively. Optimal configuration has the total power loss of $833 \mathrm{~kW}$ and the minimum bus voltage of 0.979 p.u. In
TABLE III

COMPARISON OF CONVENTIONAL AND PROPOSED SAs IN THE KEPCOS REAL DISTRIBUTION SYSTEM

\begin{tabular}{c|c|c|c|c}
\hline & Best & Average & Worst & Computation time \\
\hline \multirow{2}{*}{ Conventional SA } & $\begin{array}{r}834.51 \\
{[\mathrm{~kW}]}\end{array}$ & $\begin{array}{r}836.74 \\
{[\mathrm{~kW}]}\end{array}$ & $\begin{array}{r}839.44 \\
{[\mathrm{~kW}]}\end{array}$ & $4752[\mathrm{sec}]$ \\
\hline \multirow{2}{*}{ Proposed SA } & $\begin{array}{r}833.38 \\
{[\mathrm{~kW}]}\end{array}$ & $\begin{array}{c}834.48 \\
{[\mathrm{~kW}]}\end{array}$ & $\begin{array}{r}836.28 \\
{[\mathrm{~kW}]}\end{array}$ & $4450[\mathrm{sec}]$ \\
\hline
\end{tabular}

the implementation, good results have been reached at acceptance ratio $=0.8$, the length of Markov chain $=150, \lambda=0.4$, $\delta=1.0$, and $\varepsilon=0.3$, and $\alpha$ and $\beta$ are adjusted by the smoothing strategy. In the conventional simulated annealing, good results have been reached with the parameter $\alpha=1000.0, \beta=1000.0$, acceptance ratio $=0.95$, decrement constant $=0.95$, the length of Markov chain $=150$, and final value in stopping criterion $=$ 50. Conventional simulated annealing and the proposed simulated annealing are tested to show the effectiveness of the proposed algorithm, where the computation time of both methods is in the average of ten trails for a fair comparison.

As shown in Table III, the proposed methodology performs better than the conventional simulated annealing. The polynomial-time cooling schedule asymptotically leads to an optimal solution in the complex solution surface, and the proposed perturbation mechanism, by considering system topology and temperature, is more efficient in large-scale distribution systems. Moreover, the smoothing strategy enables simulated annealing to escape local minima. It is clear that the proposed methodology improves the convergence property and the computation time in large-scale distribution systems, and the improvement in convergence property will be more significant as the system size increases.

\section{CONCLUSION}

In this paper, we propose an improved simulated annealing algorithm for network reconfiguration in large-scale distribution systems. To improve the performance of simulated annealing, the polynomial-time cooling schedule is used which is based on the calculation of the statistics during the search. The proposed topology-based perturbation mechanism generates a network configuration related to the system topology and temperature, which allows solution space to be diversified at high temperatures and intensified at the attraction region at a lower temperature. Moreover, we use cost function with a smoothing strategy that enables the simulated annealing to escape more easily from local minima and to reach rapidly to the vicinity of an optimal solution. The proposed methodology is effective in large-scale distribution systems, and its search capability becomes more significant as the system size increases. The validity and effectiveness of the proposed methodology was demonstrated in a 148-bus system and a real distribution system of the Korea Electric Power Corporation.

\section{REFERENCES}

[1] A. Merlin and H. Back, "Search for a minimum loss operating spanning tree configuration for urban power distribution System," in Proc. 5th Power Syst. Computation Conf. (PSCC), Cambridge, U.K., 1975, Paper 1.2/6. 
[2] D. Shirmohammadi and H. W. Hong, "Reconfiguration of electric distribution networks for resistive losses reduction," IEEE Trans. Power Delivery, vol. 4, pp. 1402-1498, Apr. 1989.

[3] K. Aoki, T. Ichimori, and M. Kanezashi, "Normal state optimal load allocation in distribution systems," IEEE Trans. Power Delivery, vol. PWRD-2, pp. 147-155, Jan. 1987.

[4] S. Civanlar, J. J. Grainger, and S. H. Lee, "Distribution feeder reconfiguration for loss reduction," IEEE Trans. Power Delivery, vol. 3, pp. 1217-1223, July 1988.

[5] M. E. Baran and F. F. Wu, "Network reconfiguration in distribution systems for loss reduction and load balancing," IEEE Trans. Power Delivery, vol. 4, pp. 1401-1407, Apr. 1989.

[6] C. C. Liu, S. J. Lee, and S. S. Venkata, "An expert system optimal aid for restoration and loss reduction of distribution systems," IEEE Trans. Power Delivery, vol. 3, pp. 619-625, May 1988.

[7] H. D. Chiang and R. M. Jean-Jumeau, "Optimal network reconfiguration distribution System: Part 1: A new formulation and a solution methodology," IEEE Trans. Power Delivery, vol. 5, pp. 1902-1909, Nov. 1990.

[8] _ "Optimal network reconfigurations in distribution system: Part 2: A solution algorithm and numerical results," IEEE Trans. Power Delivery, vol. 5, pp. 1568-1574, July 1990.

[9] H. C. Chang and C. C. Kuo, "Network reconfiguration in distribution systems using simulated annealing," Elect. Power Syst. Res., vol. 29, no. 3, pp. 227-238, 1994.

[10] D. Jiang and R. Baldick, "Optimal electric distribution system switch reconfiguration and capacitor control," IEEE Trans. Power Syst., vol. 11, pp. 890-897, May 1996.

[11] C. T. Su and C. S. Lee, "Feeder reconfiguration and capacitor setting for loss reduction of distribution systems," Elect. Power Syst. Res., vol. 58, no. 2, pp. 97-102, 2001.

[12] K. Nara, A. Shiose, M. Kitagawa, and T. Ishihara, "Implementation of genetic algorithm for distribution system loss minimum reconfiguration," IEEE Trans. Power Syst., vol. 7, pp. 1044-1051, Aug. 1992.

[13] F. Glover and M. Laguna, Tabu Search. Norwell, MA: Kluwer, 1997.

[14] D. Bouchard, M. Salama, and A. Chikhani, "Survey of modern techniques for distribution feeder reconfiguration for loss minimization," Int. J. Eng. Intell. Syst. Elect. Eng. Commun., vol. 6, no. 3, pp. 173-182, Sept. 1998.

[15] K. Nara, "State of the arts of the modern heuristics application to power systems," in IEEE PES Winter Meeting, Singapore, January 2000.

[16] V. Laarhoven and E. Aarts, Simulated Annealing: Theory and Application. Holland: D. Reidel, 1987.

[17] E. Aarts and J. Korst, Simulated Annealing and Boltzman Machines. New York: Wiley, 1989.

[18] S. Kirkpatrick, C. D. Gelatto, and M. P. Vecchi, "Optimization by simulated annealing," Science, vol. 220, pp. 671-680, May 1983.

[19] V. Cerny, "Thermodynamical approach to the traveling salesman problem: An efficient simulated algorithm," J. Optim. Theory Applicat., vol. 45, no. 1, pp. 41-51, 1985.

[20] G. Wang and N. Ansari, "Searching for optimal frame patterns in an integrated TDMA communication system using mean field annealing," IEEE Trans. Neural Networks, vol. 9, pp. 1292-1300, Nov. 1998.

[21] A. H. Mantawy, Y. L. Abdel-Magid, and S. Z. Selim, "A simulated annealing algorithm for unit commitment," IEEE Trans. Power Syst., vol. 13, pp. 197-204, Feb. 1998.

[22] S. Kirkpatrick and E. Stoll, "A very fast shift-register sequence random number generator," J. Comput. Phys., vol. 40, no. 2, pp. 517-526, Apr. 1981.
Young-Jae Jeon received the B.Sc., M.E., and Ph.D. degrees in electrical engineering from Soongsil University, Seoul, Korea, in 1995, 1997, and 2001, respectively.

His current research interests include optimization and neural networks in power systems.

Dr. Jeon is a member of KIEE.

Jae-Chul Kim (M'88) received the B.S.E.E. degree in electrical engineering from Soongsil University, Seoul, Korea, in 1979, and the M.Sc. and Ph.D. degrees in electrical engineering from Seoul National University, Seoul, Korea, in 1983 and 1987, respectively.

Since 1988, he has been a Professor of electrical engineering at Soongsil University. His areas of interest are power system stability, protective relays, distribution automation systems, and diagnosis of power system equipment.

Dr. Kim is a member of KIEE.

Jin-O. Kim (M'94) received the B.Sc. and M.Sc. degrees in electrical engineering from Seoul National University, Seoul, Korea, in 1980 and 1983, respectively, and Ph.D. in electrical engineering from Texas A\&M University, College Station, in 1991.

Currently, he is an Associate Professor of Electrical Engineering at Hanyang University, Seoul, Korea. His current research interests include computer modeling and simulation of power systems using AI, power system reliability, and power quality.

Joong-Rin Shin (M'90) received the B.Sc., M.Sc., and Ph.D. degrees in electrical engineering from Seoul National University, Seoul, Korea, in 1977, 1984, and 1989 , respectively.

Currently, he is a Professor of electrical engineering at Kon-Kuk University, Seoul, where he has been since 1990. His major interests are power system analysis, power system planning and reliability evaluation, and power system engineering tools. From 1977 to 1990, he was an engineer with the Korea Electric Power Corporation.

Kwang Y. Lee (F'01) received the B.Sc. degree in electrical engineering from Seoul National University, Seoul, Korea, in 1964, and the M.Sc. degree in electrical engineering from North Dakota State University, Fargo, in 1968. He received the Ph.D. degree in system science from Michigan State University, East Lansing, in 1971

Currently, he is Professor of electrical engineering at Pennsylvania State University (PSU), University Park. He has also been on the faculties of Michigan State University, Oregon State University, and University of Houston, Houston, TX. He is currently in charge of the Power Engineering Program and the Power System Control Laboratory at PSU. His interests are power systems control, operation and planning, and intelligent system applications.

Dr. Lee is a registered Professional Engineer. 